\title{
What happens after activation of ascaridole? Reactive compounds and their implications for skin sensitization.
}

\author{
Amar G. Chittiboyina, ${ }^{\S, *}$ Cristina Avonto, ${ }^{\S}$ and Ikhlas A. Khan. ${ }^{\S, \varnothing}$ \\ ${ }^{5}$ National Center for Natural Products Research; " Department of Pharmacognosy, Division of \\ BioMolecular Sciences; School of Pharmacy, University of Mississippi, University, MS 38677, USA. \\ amar@olemiss.edu \\ SUPPORTING INFORMATION
}

Contents

Page\#

1. Figure S1. ${ }^{1} \mathrm{H}$ NMR $(500 \mathrm{MHz}$, Chloroform-d) of ascaridole. 2

2. Figure S2. ${ }^{13} \mathrm{C}$ NMR $(125 \mathrm{MHz}$, Chloroform-d) of ascaridole 3

3. Figure S3. Arrayed ${ }^{1}$ HNMR spectra for Ascaridole and DCYA, before addition of DBN $\quad 4$

4. Table S1. Select normalized integral values of DCYA and Ascaridole before addition of DBN 5

5. Figure S4. Arrayed ${ }^{1}$ HNMR spectra for Ascaridole and DCYA, after addition of DBN 6

6. Table S2. Select normalized integral values of DCYA and Ascaridole after addition of DBN) 7

7. Figure S5. Structure of isolated compounds $\mathbf{2 e}, \mathbf{2 b}, \mathbf{2 d}, \mathbf{2 c} \quad 8$

8. Table S3. ${ }^{1} \mathrm{H}$ and ${ }^{13} \mathrm{C}$ assignments of isolated ascaridole by-products $\mathbf{2 e}, \mathbf{2 b}, \mathbf{2 d}, \mathbf{2 c} \quad 8$

8. Figure S6. ${ }^{1} \mathrm{H} \mathrm{NMR}$ in $\mathrm{CDCl}_{3}(500 \mathrm{MHz})$ of compound $2 \mathrm{e}$

9. Figure S7. ${ }^{13} \mathrm{C} \mathrm{NMR}$ in $\mathrm{CDCl}_{3}(125 \mathrm{MHz})$ of compound $2 \mathbf{e} . \quad 10$

10. Figure S8. TLC of activated ascaridole mixture after trapping experiment with DCYA 11

11. Figure S9. LC-MS of activated ascaridole mixture after treating with DCYA in pH10 buffer 11

12. Table S9. ${ }^{1} \mathrm{H}$ and ${ }^{13} \mathrm{C}$ assignments of isolated diastereomeric DCYA adducts. 13

13. Figure S10. ${ }^{1} \mathrm{H}$ NMR in CDOD $(500 \mathrm{MHz})$ of adduct $1 . \quad 14$

13. Figure S11. ${ }^{13} \mathrm{C}$ NMR in CDOD $(125 \mathrm{MHz})$ of adduct $1 . \quad 15$

13. Figure S12. ${ }^{1} \mathrm{H}$ NMR in CDOD $(500 \mathrm{MHz})$ of adduct $2 . \quad 16$

13. Figure S13. ${ }^{13} \mathrm{C}$ NMR in CDOD $(125 \mathrm{MHz})$ of adduct $2 . \quad 17$ 
Figure S1. ${ }^{1} \mathrm{H}$ NMR (500 MHz, Chloroform-d) of ascaridole. $\delta 6.48(\mathrm{~d}, J=8.5 \mathrm{~Hz}, 1 \mathrm{H}), 6.40(\mathrm{~d}, J=8.5 \mathrm{~Hz}, 1 \mathrm{H}), 2.05-1.97(\mathrm{~m}, 2 \mathrm{H}), 1.91($ hept, $J=$ $7.4,6.9 \mathrm{~Hz}, 1 \mathrm{H}), 1.53-1.47(\mathrm{~m}, 2 \mathrm{H}), 1.36(\mathrm{~s}, 3 \mathrm{H}), 0.98(\mathrm{~d}, J=7.0 \mathrm{~Hz}, 6 \mathrm{H})$.

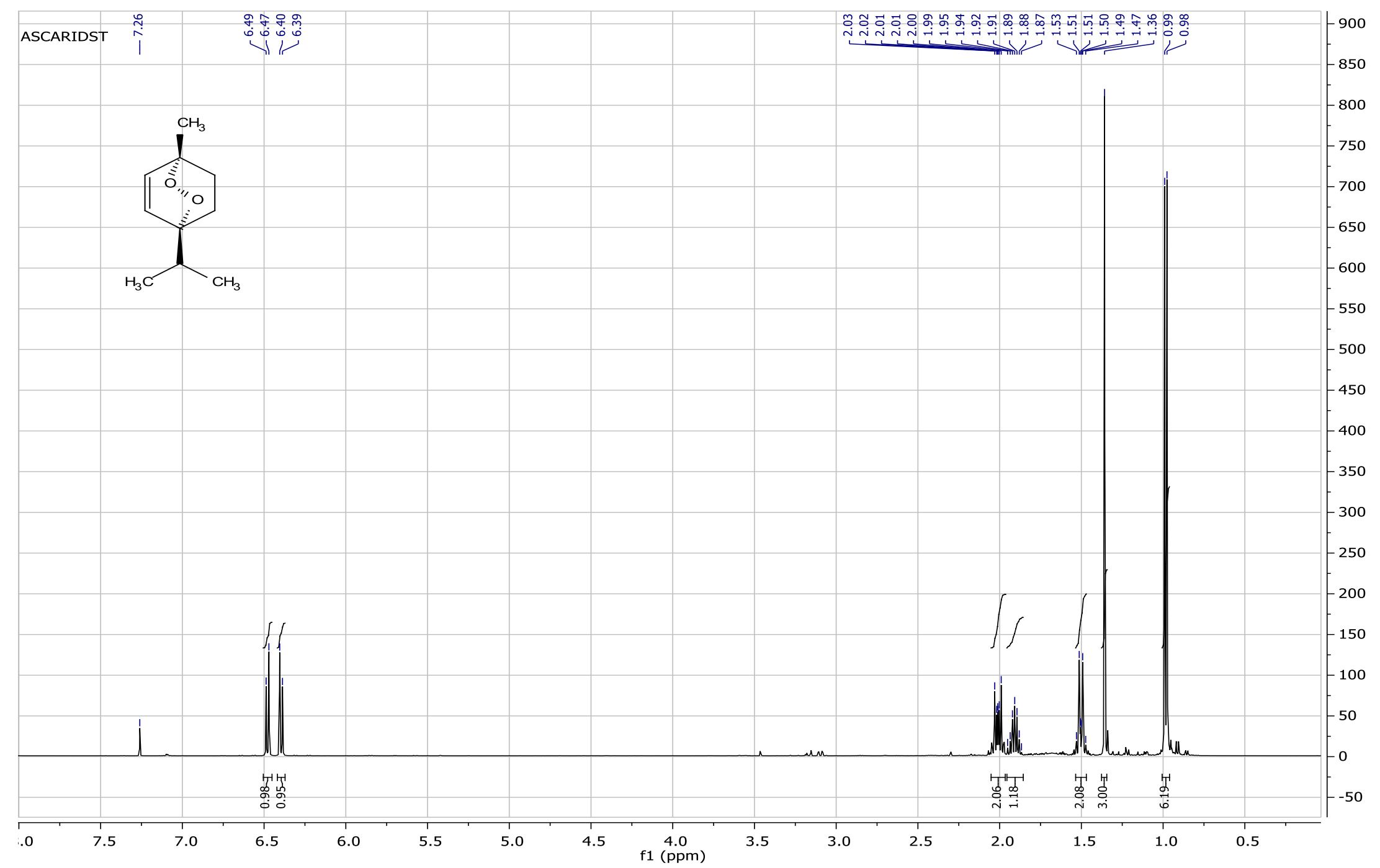


Figure S2. ${ }^{13} \mathrm{C}$ NMR $(125 \mathrm{MHz}$, Chloroform-d) of ascaridole. $\delta$ 136.5, 133.1, 79.9, 74.5, 32.2, 29.6, 25.7, 21.5, 17.3, 17.3.

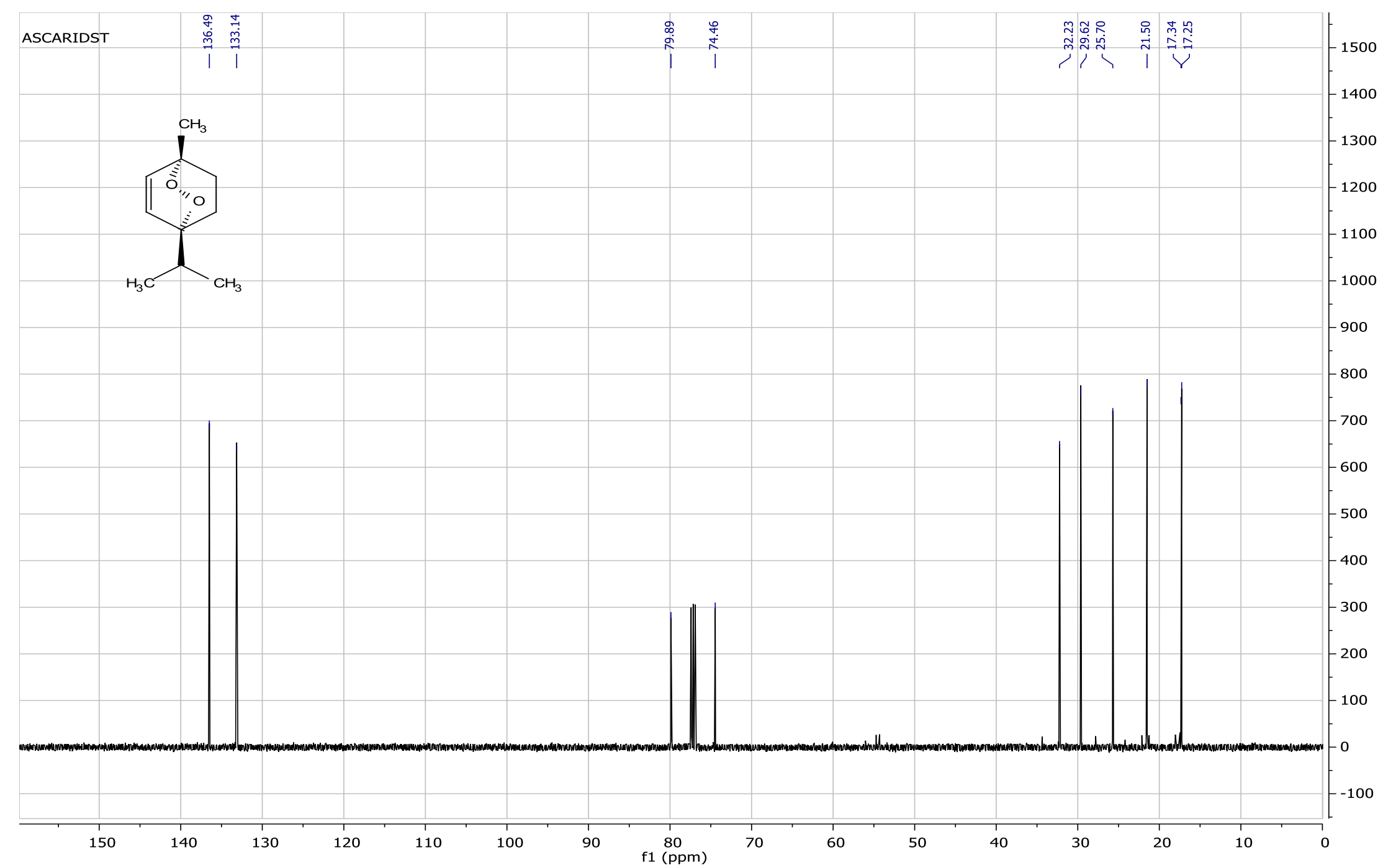


Figure S3. Arrayed ${ }^{1}$ HNMR spectra for Ascaridole and DCYA, before addition of DBN

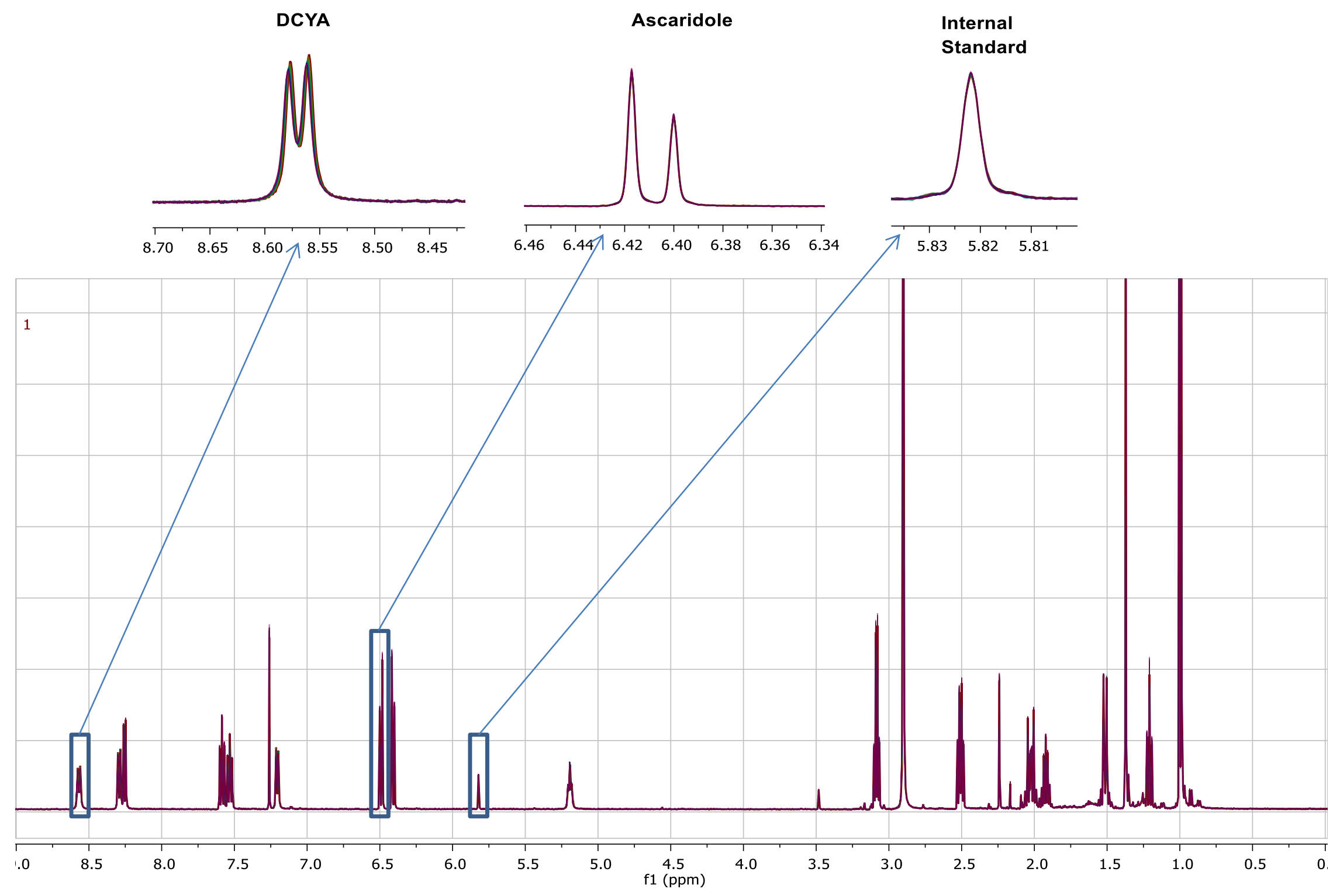


Table S1. Select normalized integral values of DCYA and Ascaridole before addition of DBN (2,5-dimethylfuran was used as internal standard).

\begin{tabular}{|c|c|c|c|c|}
\hline Time (min) & DCYA-Peak1 & DCYA-Peak2 & Ascaridole-Peak1 & Ascaridole-Peak2 \\
\hline$X(I)$ & $Y(X)$ & $\mathrm{Y} 2(\mathrm{X})$ & Y3(X) & $\mathrm{Y} 4(\mathrm{X})$ \\
\hline ARR_DATA(I) & Integral(8.620,8.520) & Integral(7.230,7.180) & Integral(6.520,6.460) & Integral(6.440,6.380) \\
\hline 0 & 100.00 & 100.00 & 100.00 & 100.00 \\
\hline 3 & 99.79 & 100.37 & 100.41 & 100.41 \\
\hline 7 & 99.79 & 100.50 & 100.23 & 100.54 \\
\hline 10 & 99.71 & 100.54 & 100.34 & 100.53 \\
\hline 13 & 99.47 & 100.75 & 100.11 & 100.14 \\
\hline 17 & 99.16 & 99.94 & 99.26 & 99.67 \\
\hline 20 & 101.04 & 101.60 & 100.79 & 100.97 \\
\hline 23 & 99.30 & 100.21 & 99.25 & 99.26 \\
\hline 27 & 100.11 & 100.60 & 99.79 & 100.02 \\
\hline 30 & 101.11 & 101.97 & 101.08 & 101.41 \\
\hline 33 & 101.57 & 101.94 & 100.68 & 100.85 \\
\hline 36 & 99.90 & 100.87 & 99.44 & 99.66 \\
\hline 40 & 99.74 & 100.18 & 99.15 & 99.33 \\
\hline 43 & 100.70 & 101.21 & 100.23 & 100.45 \\
\hline 46 & 99.82 & 100.55 & 99.29 & 99.38 \\
\hline 50 & 100.29 & 101.31 & 99.81 & 100.17 \\
\hline 53 & 100.29 & 100.09 & 99.08 & 99.28 \\
\hline 56 & 100.20 & 100.78 & 99.60 & 99.64 \\
\hline 60 & 100.04 & 100.49 & 99.51 & 99.60 \\
\hline 63 & 101.06 & 101.53 & 100.27 & 100.59 \\
\hline
\end{tabular}


Figure S4. Arrayed ${ }^{1}$ HNMR spectra for Ascaridole and DCYA, after addition of DBN.

$$
\text { DCYA Ascaridole }
$$

Internal

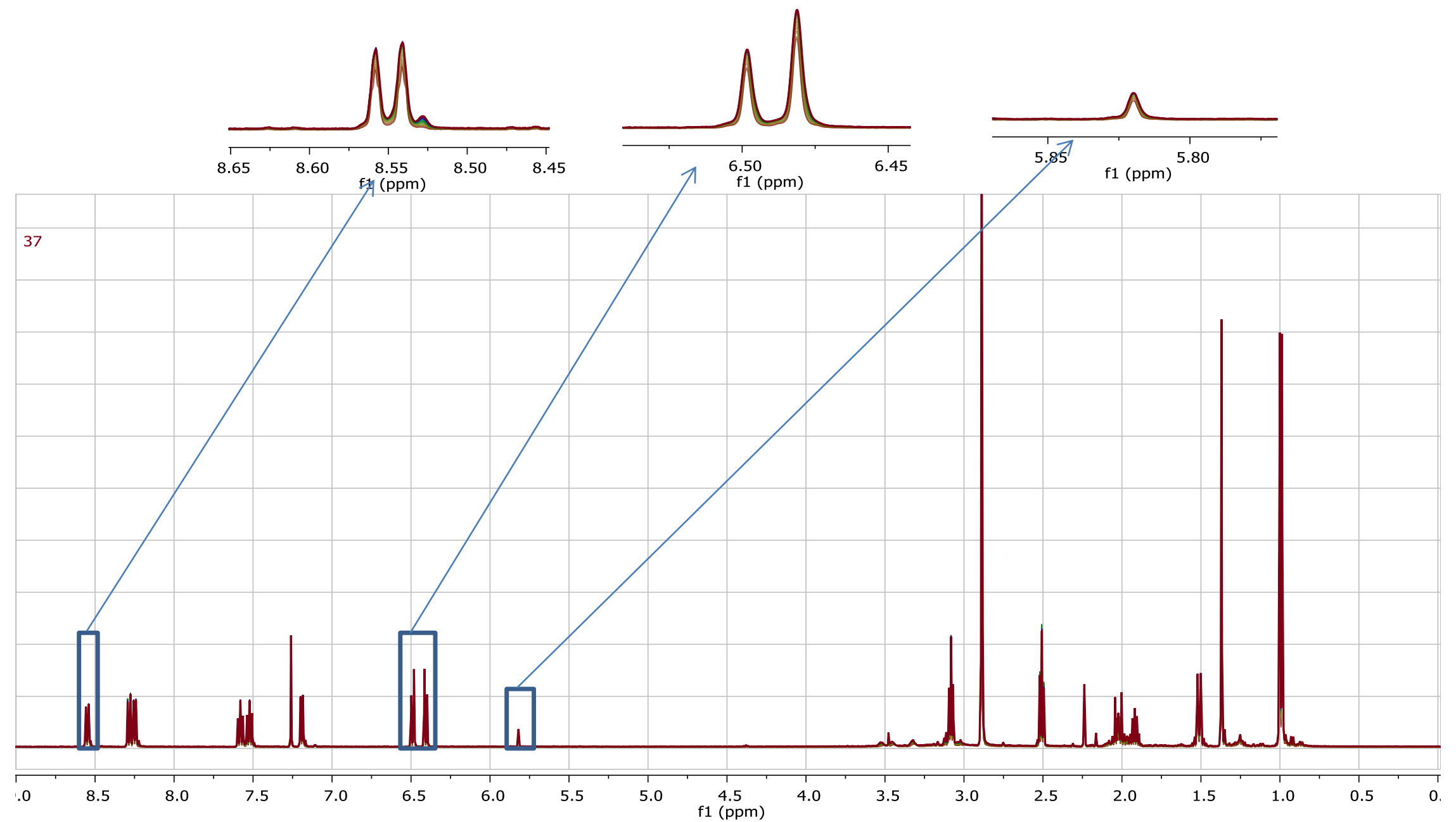


Table S2. Select normalized integral values of DCYA and Ascaridole after addition of DBN (2,5dimethylfuran was used as internal standard).

\begin{tabular}{|c|c|c|c|c|}
\hline Time (min) & DCYA-Peak1 & DCYA-Peak2 & Ascaridole-Peak1 & Ascaridole-Peak2 \\
\hline$X(1)$ & $Y(X)$ & $\mathrm{Y} 1(\mathrm{X})$ & $\mathrm{Y} 3(\mathrm{X})$ & $\mathrm{Y} 4(\mathrm{X})$ \\
\hline ARR_DATA(I) & Integral(8.620,8.520) & Integral(7.230,7.180) & Integral(6.520,6.460) & Integral(6.440,6.380) \\
\hline 0 & 100 & 100 & 100 & 100 \\
\hline 3 & 100.93 & 101.36 & 101.01 & 100.88 \\
\hline 7 & 100.89 & 100.54 & 100.80 & 100.20 \\
\hline 10 & 100.93 & 100.38 & 101.27 & 100.87 \\
\hline 13 & 101.46 & 101.50 & 102.20 & 101.86 \\
\hline 17 & 100.59 & 100.30 & 101.36 & 101.15 \\
\hline 20 & 100.79 & 99.95 & 100.90 & 100.74 \\
\hline 23 & 100.12 & 99.10 & 100.17 & 99.96 \\
\hline 27 & 100.72 & 99.58 & 100.79 & 100.98 \\
\hline 30 & 100.16 & 98.70 & 100.26 & 99.92 \\
\hline 33 & 100.39 & 99.31 & 100.95 & 100.69 \\
\hline 36 & 100.95 & 99.32 & 101.20 & 101.23 \\
\hline 40 & 100.31 & 98.42 & 100.71 & 100.51 \\
\hline 43 & 100.68 & 98.71 & 100.80 & 100.68 \\
\hline 46 & 100.04 & 97.93 & 100.50 & 100.25 \\
\hline 50 & 100.64 & 98.52 & 101.24 & 100.89 \\
\hline 53 & 100.21 & 97.49 & 99.99 & 100.14 \\
\hline 56 & 100.88 & 98.56 & 101.31 & 101.32 \\
\hline 60 & 100.80 & 98.59 & 101.79 & 101.43 \\
\hline 63 & 100.59 & 97.83 & 101.03 & 100.62 \\
\hline 66 & 100.69 & 98.05 & 101.29 & 101.11 \\
\hline 70 & 100.67 & 97.57 & 100.92 & 100.85 \\
\hline 73 & 101.37 & 98.10 & 102.08 & 101.81 \\
\hline 76 & 99.64 & 96.10 & 99.97 & 99.82 \\
\hline 80 & 101.10 & 97.85 & 101.77 & 101.32 \\
\hline 83 & 99.97 & 96.83 & 100.77 & 100.28 \\
\hline 86 & 100.99 & 97.48 & 101.50 & 100.87 \\
\hline 90 & 99.75 & 95.88 & 100.17 & 99.87 \\
\hline 93 & 100.88 & 96.65 & 101.34 & 100.95 \\
\hline 96 & 101.43 & 97.48 & 102.00 & 101.58 \\
\hline 100 & 100.94 & 97.10 & 101.61 & 101.31 \\
\hline 103 & 100.74 & 96.39 & 101.15 & 101.00 \\
\hline 106 & 100.70 & 96.33 & 100.80 & 100.40 \\
\hline 109 & 101.33 & 96.76 & 101.57 & 101.31 \\
\hline 113 & 100.24 & 95.69 & 100.58 & 100.25 \\
\hline 116 & 101.93 & 97.27 & 102.51 & 102.04 \\
\hline 119 & 102.15 & 97.38 & 102.59 & 102.09 \\
\hline
\end{tabular}


Figure S5. Structure of isolated compounds $\mathbf{2 e}, \mathbf{2 b}, \mathbf{2 d}, \mathbf{2 c}$<smiles>[3H]C1(O)C=CC(=O)CC1</smiles>

$2 e$<smiles>CC(C)C1=CCC2(C(C)C)OC1[C@H]2O</smiles>

2b<smiles>CC(C)C12CCC(O)(O)C(O1)[C@H](O)C2</smiles>

2d<smiles>CC(C)[C@]1(O)CC[C@@](C)(O)[C@H](O)[C@@H]1O</smiles>

2c

Table S3. ${ }^{1} \mathrm{H}$ and ${ }^{13} \mathrm{C}$ assignments of isolated ascaridole by-products $2 \mathrm{c}-2 \mathrm{e}$

$2 \mathrm{e}$

$2 b$ 2d

\begin{tabular}{|c|c|c|c|c|c|c|c|c|}
\hline Pos. & $\begin{array}{l}\delta_{H}(\text { mult }, J \\
\text { in } \mathrm{Hz})\end{array}$ & $\delta_{c}$ & $\begin{array}{l}\delta_{H} \text { (mult, } J \\
\text { in } H z \text { ) }\end{array}$ & $\delta_{c}$ & $\delta_{H}($ mult, $J$ in $H z)$ & $\delta_{c}$ & $\delta_{H}($ mult, $J$ in $H z)$ & $\delta_{c}$ \\
\hline 1 & - & 199.3 & - & 131.9 & - & 70.6 & - & 84.8 \\
\hline 2 & $\begin{array}{l}5.85(\mathrm{dd}, 10.1 \text {, } \\
2.5)\end{array}$ & 128.0 & 4.18 (bs) & 68.2 & $3.56(\mathrm{~m})$ & 71.2 & $3.58(\mathrm{dt}, 9.0,1.8)$ & 73.6 \\
\hline 3 & $6.75(d, 10.1)$ & 155.1 & $3.29(\mathrm{~d}, 2.9)$ & 61.1 & $3.19(\mathrm{dd}, 4.8,1.0)$ & 59.8 & $3.78(\mathrm{dt}, 9.1,1.8)$ & 70.5 \\
\hline 4 & - & 68.5 & - & 66.0 & - & 68.2 & - & 91.4 \\
\hline $5 a$ & $2.10^{a}(\mathrm{~m})$ & 37.3 & $2.35(\mathrm{~m})$ & 25.3 & $1.99(\mathrm{~m})$ & 19.8 & $2.00^{a}(\mathrm{~m})$ & 25.2 \\
\hline $5 b$ & $2.10^{a}(\mathrm{~m})$ & 37.3 & $2.26(\mathrm{~m})$ & 25.3 & $\begin{array}{l}1.78 \text { (dddd, 14.9, } \\
5.5,3.2,1.0)\end{array}$ & 19.8 & $1.41(\mathrm{~m})$ & 25.2 \\
\hline $6 a$ & $2.60(\mathrm{~m})$ & 35.0 & $\begin{array}{l}5.23(\mathrm{ddt}, 4.2 \\
2.8,1.4)\end{array}$ & 119.6 & $\begin{array}{l}1.50 \text { (ddd, 14.1, } \\
11.2,5.5)\end{array}$ & 28.0 & $2.00^{a}(\mathrm{~m})$ & 29.3 \\
\hline $6 b$ & $\begin{array}{l}2.39 \text { (dddd, } \\
17.3,9.3,5.6 \\
2.5)\end{array}$ & 35.0 & - & - & $\begin{array}{l}1.30 \text { (dddd, 14.0, } \\
5.5,3.4,1.7)\end{array}$ & 28.0 & $1.30(\mathrm{~m})$ & 29.3 \\
\hline 7 & $1.44(\mathrm{~d}, 2.4)$ & 27.2 & $\begin{array}{l}1.60 \text { (hept, } \\
7.0 \text { ) }\end{array}$ & 34.5 & 1.57 (hept, 7.0) & 34.7 & $1.99^{a}(\mathrm{~m})$ & 32.9 \\
\hline 8 & - & - & $0.99(d, 6.9)$ & 17.7 & $0.97^{a}(\mathrm{dd}, 6.9)$ & 18.3 & $0.99^{*}(d, 6.9)$ & 17.8 \\
\hline 9 & - & - & $0.95(d, 6.9)$ & 18.5 & $0.97^{a}(\mathrm{dd}, 6.9)$ & 17.7 & $0.98^{*}(\mathrm{~d}, 6.9)$ & 17.7 \\
\hline 10 & - & - & $1.75(\mathrm{~s})$ & 19.3 & $1.23(\mathrm{~s})$ & 26.0 & $1.38(\mathrm{~s})$ & 20.2 \\
\hline
\end{tabular}

* Inter-changeable ${ }^{a}$ Overlapped with other signals 
Figure S6. ${ }^{1} \mathrm{H} \mathrm{NMR} \mathrm{in} \mathrm{CDCl}_{3}(500 \mathrm{MHz})$ of compound $2 \mathrm{e}$.

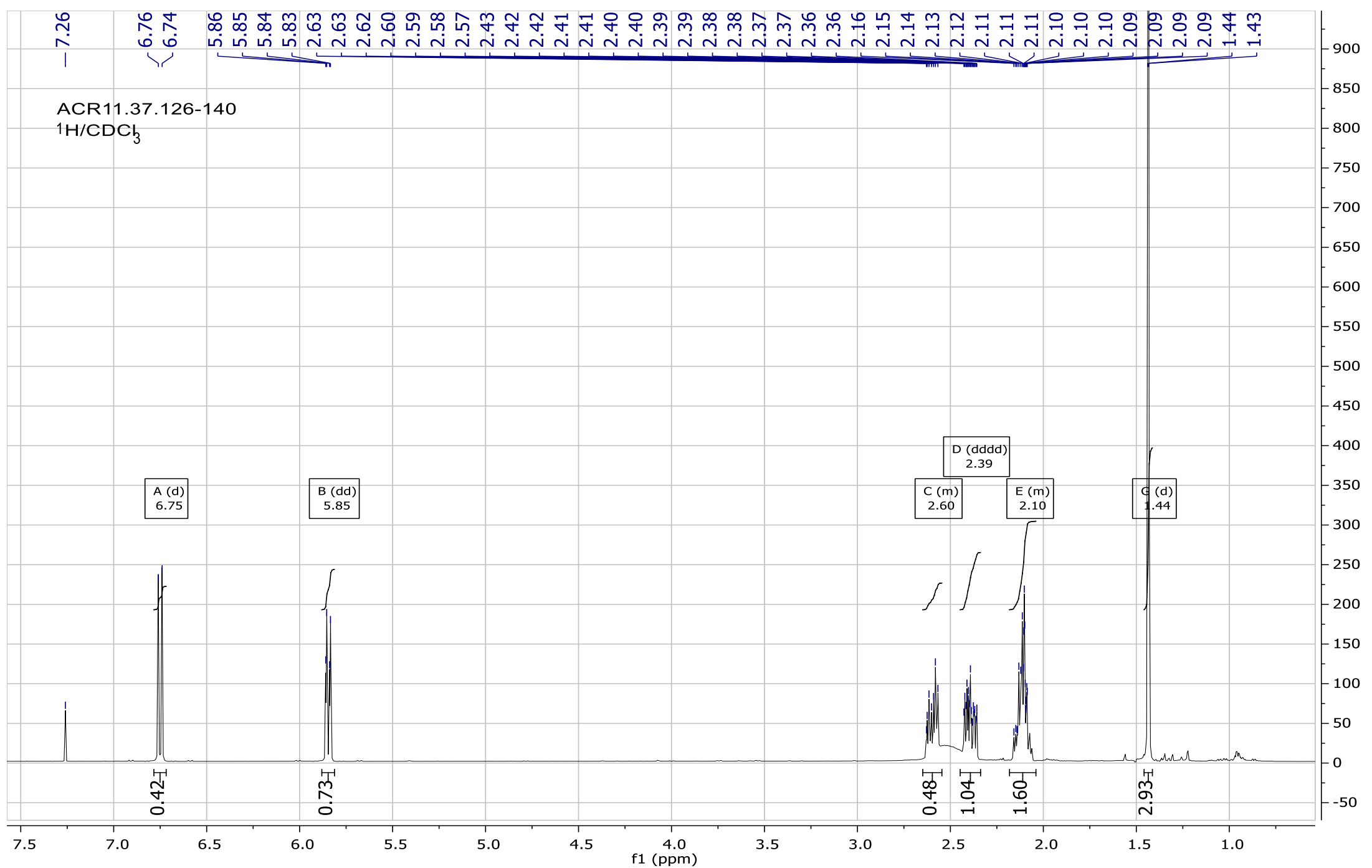


Figure S7. ${ }^{13} \mathrm{C} \mathrm{NMR}$ in $\mathrm{CDCl}_{3}(500 \mathrm{MHz})$ of compound $2 \mathbf{e}$.

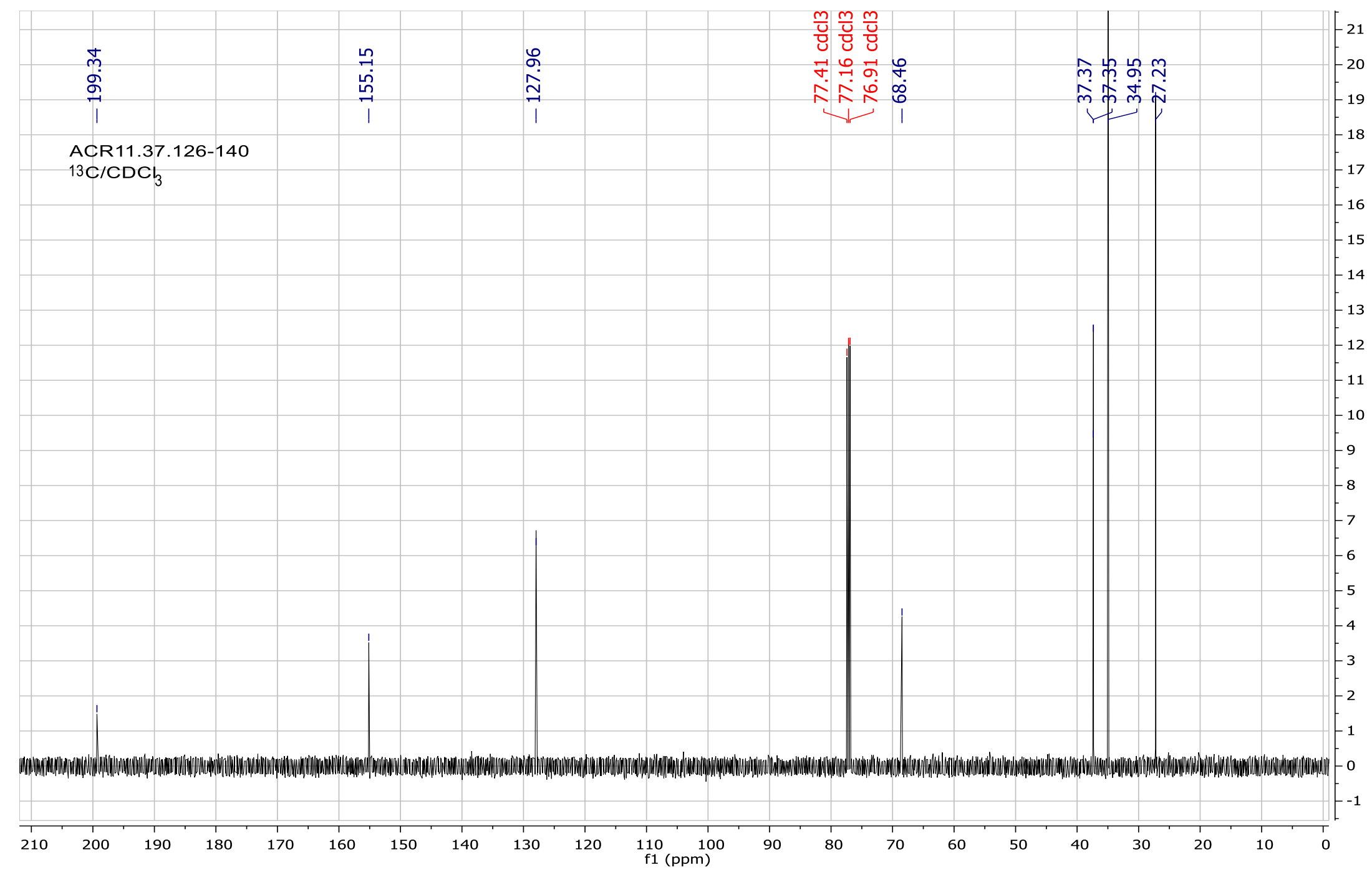


Figure S8. TLC of activated ascaridole mixture after trapping experiment with DCYA

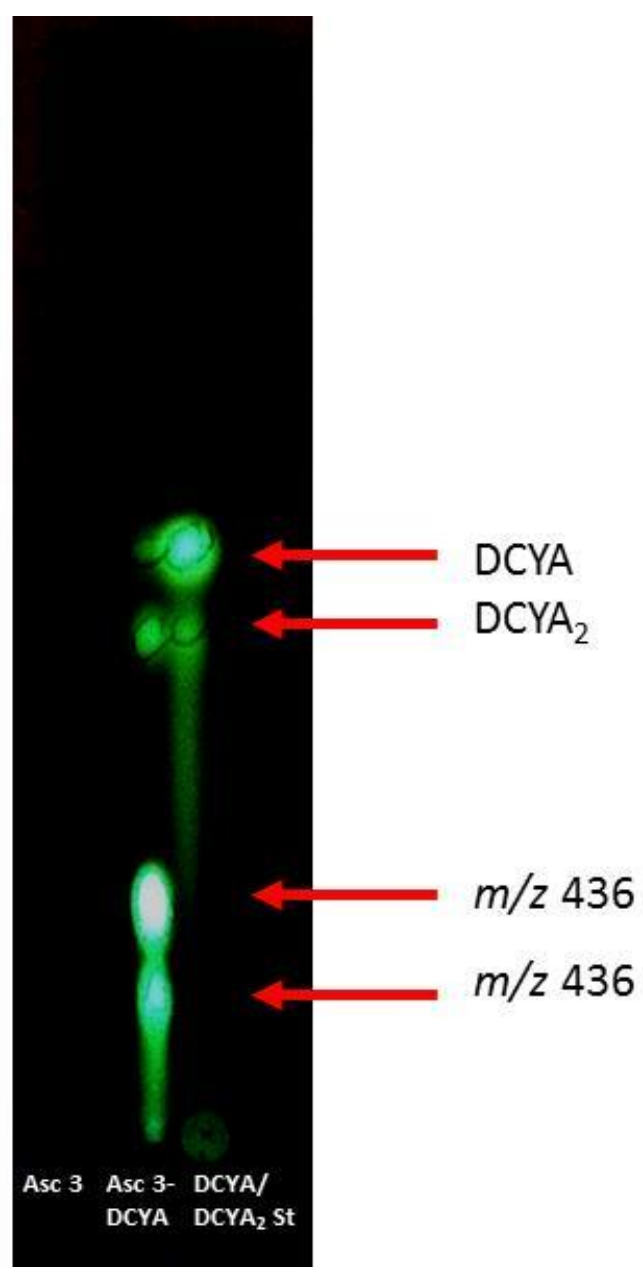

Figure S9. LC-MS of activated ascaridole mixture after treating with DCYA in pH10 buffer.

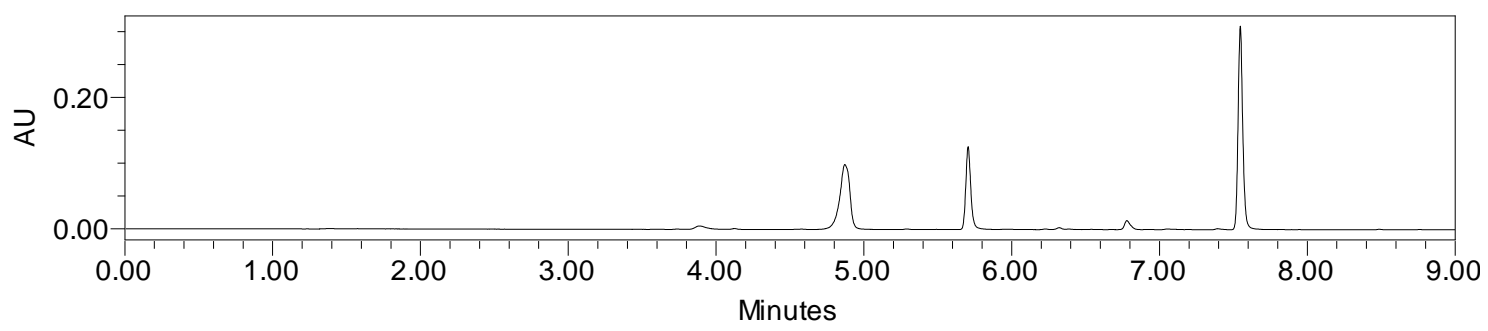

Conditions: Column: BEH Shield RP18 1.7 um, $100 \times 2.1 \mathrm{~mm}$. Column Temp: $35^{\circ} \mathrm{C}$; Sample Temp: $15{ }^{\circ} \mathrm{C}$; $U V=190-500$ nm; MS Parameters: Capillary Voltage (kV): 3.0; Cone (V): 30 (pos) and 30 (neg); Extractor 
(V): 3.0; Rf Lens (V): 0.5; Source Temp. $150^{\circ} \mathrm{C}$ ); Desolvation Temp. $350{ }^{\circ} \mathrm{C}$; Desolvation (L/hr): 650 ; Cone (L/hr): 25 and MS Range: $m / z=100-1000$
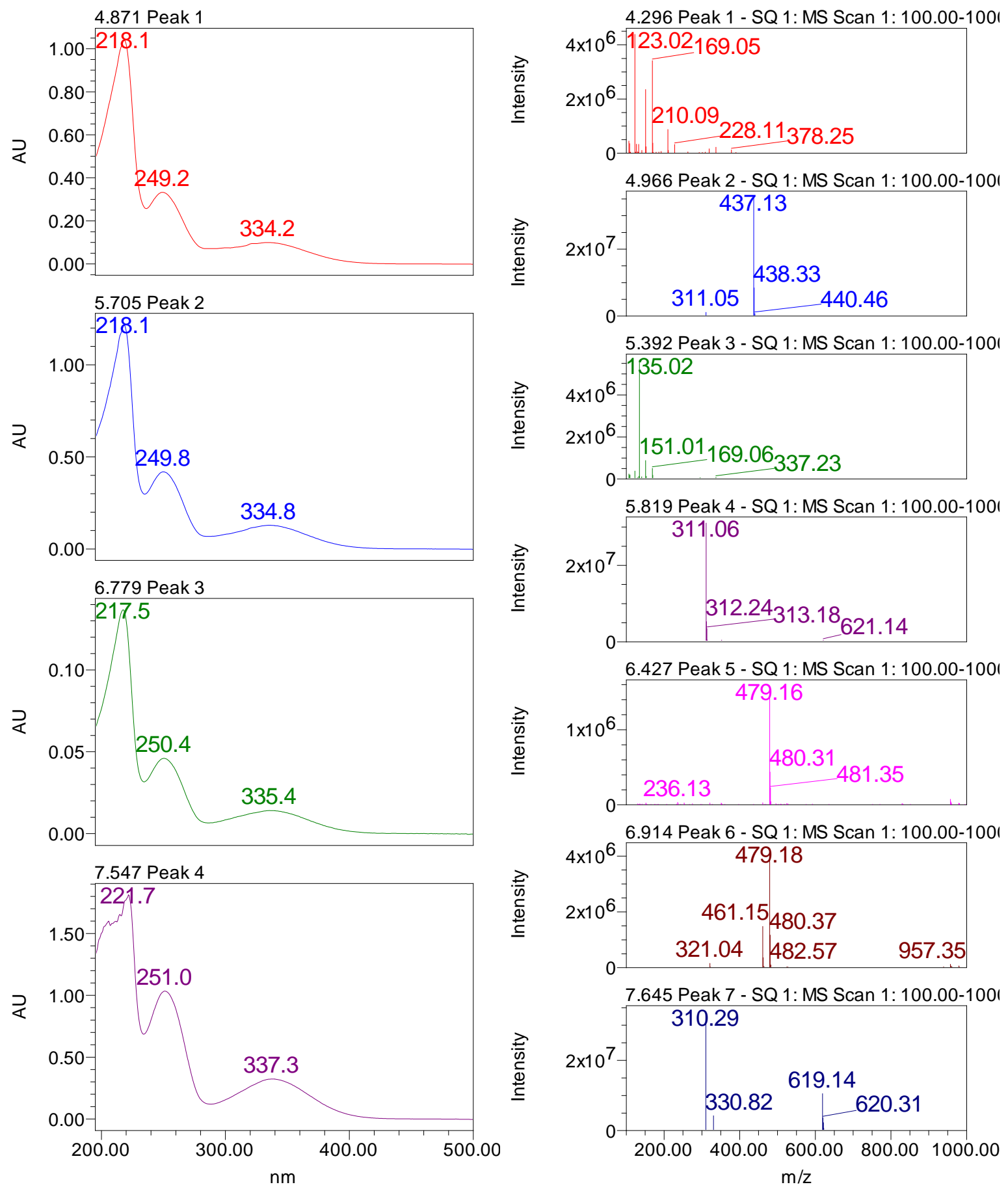
Table S9. ${ }^{1} \mathrm{H}$ and ${ }^{13} \mathrm{C}$ assignments of isolated DCYA adducts.

Adduct 1

\begin{tabular}{|c|c|c|c|c|}
\hline Pos. & $\begin{array}{l}\delta_{H} \text { (mult,J } \\
\text { in } \mathrm{Hz} \text { ) }\end{array}$ & ${ }^{13} \mathrm{C}$ & $\begin{array}{l}\delta_{H} \text { (mult,J } \\
\text { in } \mathrm{Hz} \text { ) }\end{array}$ & ${ }^{13} \mathrm{C}$ \\
\hline 1 & - & 153.2 & - & 153.2 \\
\hline 2 & $7.28(\mathrm{dd}, 7.5,0.9)$ & 116.5 & $7.26(\mathrm{dd}, 7.5,1.0)$ & 116.5 \\
\hline 3 & $7.59^{a}(\mathrm{dd}, 8.7,7.4)$ & 129.2 & $7.59^{a}(\mathrm{dd}, 8.7,7.5)$ & 129.2 \\
\hline 4 & $8.33(\mathrm{dt}, 8.7,0.9)$ & 120.6 & $8.34(\mathrm{dt}, 8.7,1.0)$ & 120.5 \\
\hline 5 & - & 137.1 & - & 137.1 \\
\hline 6 & $8.21(\mathrm{dd}, 7.3,1.1)$ & 130.2 & $8.21(\mathrm{dd}, 7.3,1.3)$ & 130.2 \\
\hline 7 & $7.57^{a}(\mathrm{dd}, 8.6,7.3)$ & 124.3 & $7.57^{a}(\mathrm{dd}, 8.6,7.3)$ & 124.3 \\
\hline 8 & $8.57(\mathrm{dt}, 8.6,1.1)$ & 131.3 & $8.56(\mathrm{dt}, 8.6,1.2)$ & 131.3 \\
\hline 9 & - & $131.3^{*}$ & - & $131.2^{*}$ \\
\hline 10 & - & $130.9 *$ & - & $130.9 *$ \\
\hline 11 & $2.88^{*}(\mathrm{~s})$ & 45.8 & $2.87^{*}(\mathrm{~s})$ & 45.8 \\
\hline 12 & $2.88 *(\mathrm{~s})$ & 45.8 & $2.87^{*}(\mathrm{~s})$ & 45.8 \\
\hline 13 & $2.51^{a}$ & 32.8 & $2.47(d t, 7.1,3.16)$ & 32.6 \\
\hline 14 & $3.03(t, 7.1)$ & 44.0 & $3.03(t, 7.1)$ & 44.4 \\
\hline 15 & - & 211.6 & - & 211.6 \\
\hline $16 a$ & $2.92^{a}(14.5,9.9,6.3,1.1)$ & 44.7 & $2.61^{a}(\mathrm{~m})$ & 46.2 \\
\hline $16 b$ & $2.23^{a}(\mathrm{ddt}, 14.5,5.6,1.8)$ & 44.7 & $2.35(\mathrm{dd}, 10.1,2.2)$ & 46.2 \\
\hline 17 & $2.90^{a}$ & 54.3 & $2.61^{a}(\mathrm{~m})$ & 54.4 \\
\hline 18 & - & 72.0 & - & 71.2 \\
\hline $19 b$ & $1.75(\mathrm{dtd}, 13.9,6.3,1.8)$ & 36.6 & 1.97 (ddd, $14.0,6.4,3.2)$ & 38.8 \\
\hline $19 a$ & $1.92(\mathrm{ddd}, 13.9,9.9,5.6 \mathrm{~Hz})$ & 36.6 & $1.66(t d, 13.6,5.0)$ & 38.8 \\
\hline $20 a$ & $2.50^{a}(\mathrm{~m})$ & 38.1 & $2.59^{a}(\mathrm{~m})$ & 37.7 \\
\hline $20 b$ & $2.17^{a}(\mathrm{~m})$ & 38.1 & $2.12(\mathrm{~m})$ & 37.7 \\
\hline 21 & $1.29(\mathrm{~s})$ & 25.8 & $1.26(\mathrm{~s})$ & 27.9 \\
\hline
\end{tabular}

\section{Adduct 2}


Figure S10. ${ }^{1} \mathrm{H}$ NMR in CDOD (500 MHz) of adduct 1.

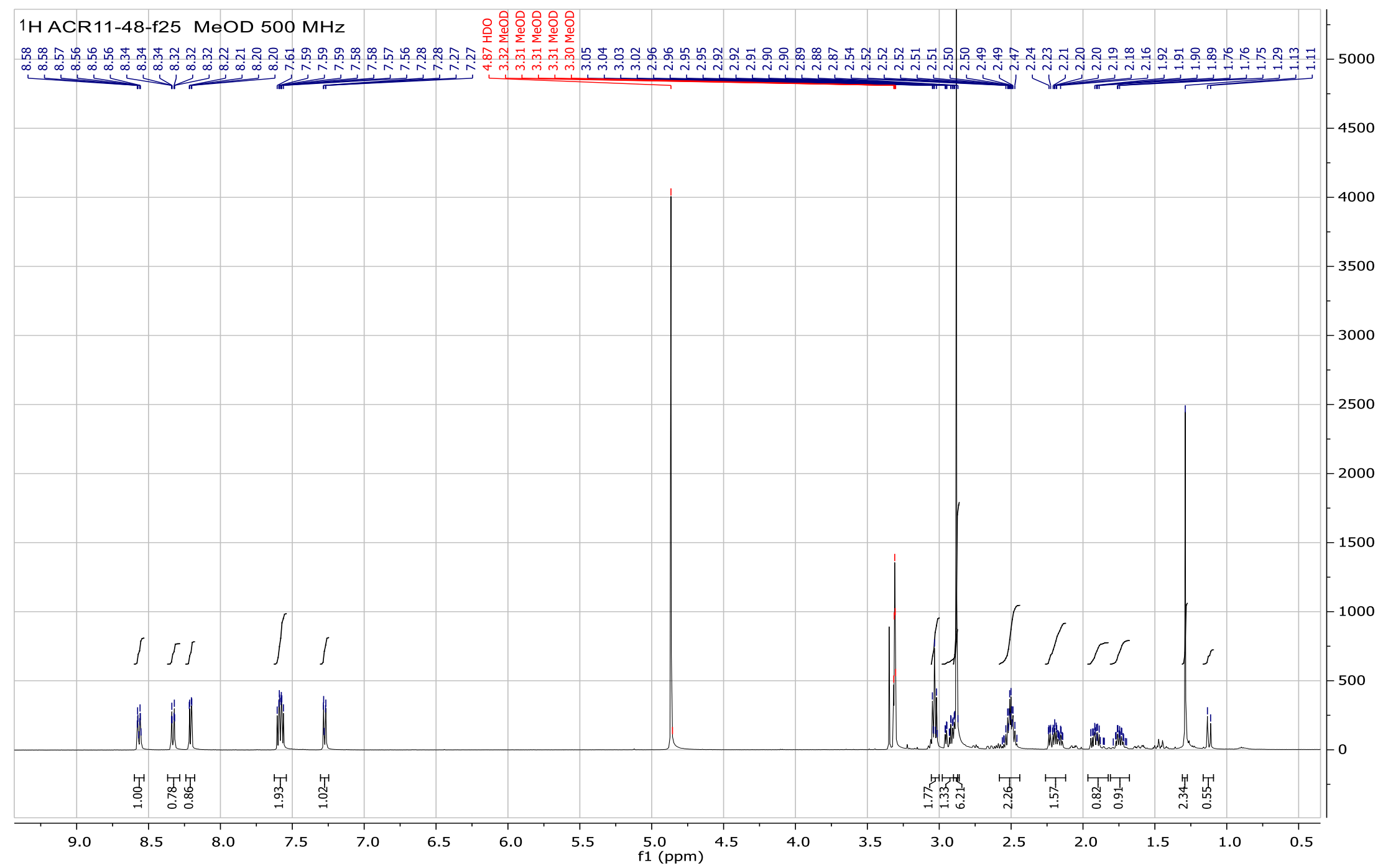


Figure S11. ${ }^{13} \mathrm{C}$ NMR in CDOD (125 MHz) of adduct 1.

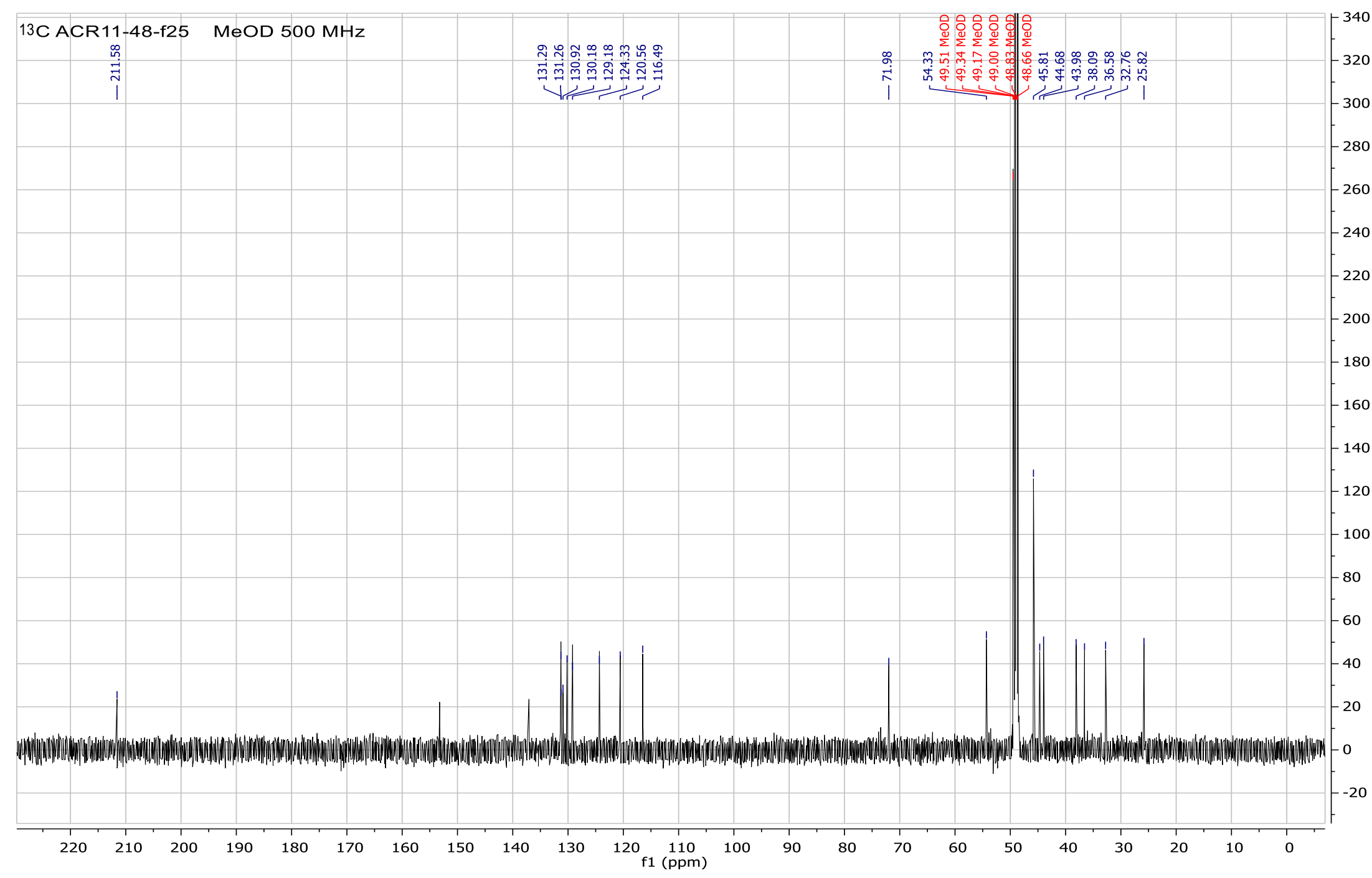


Figure S12. ${ }^{1} \mathrm{H}$ NMR in CDOD $(500 \mathrm{MHz})$ of adduct 2.

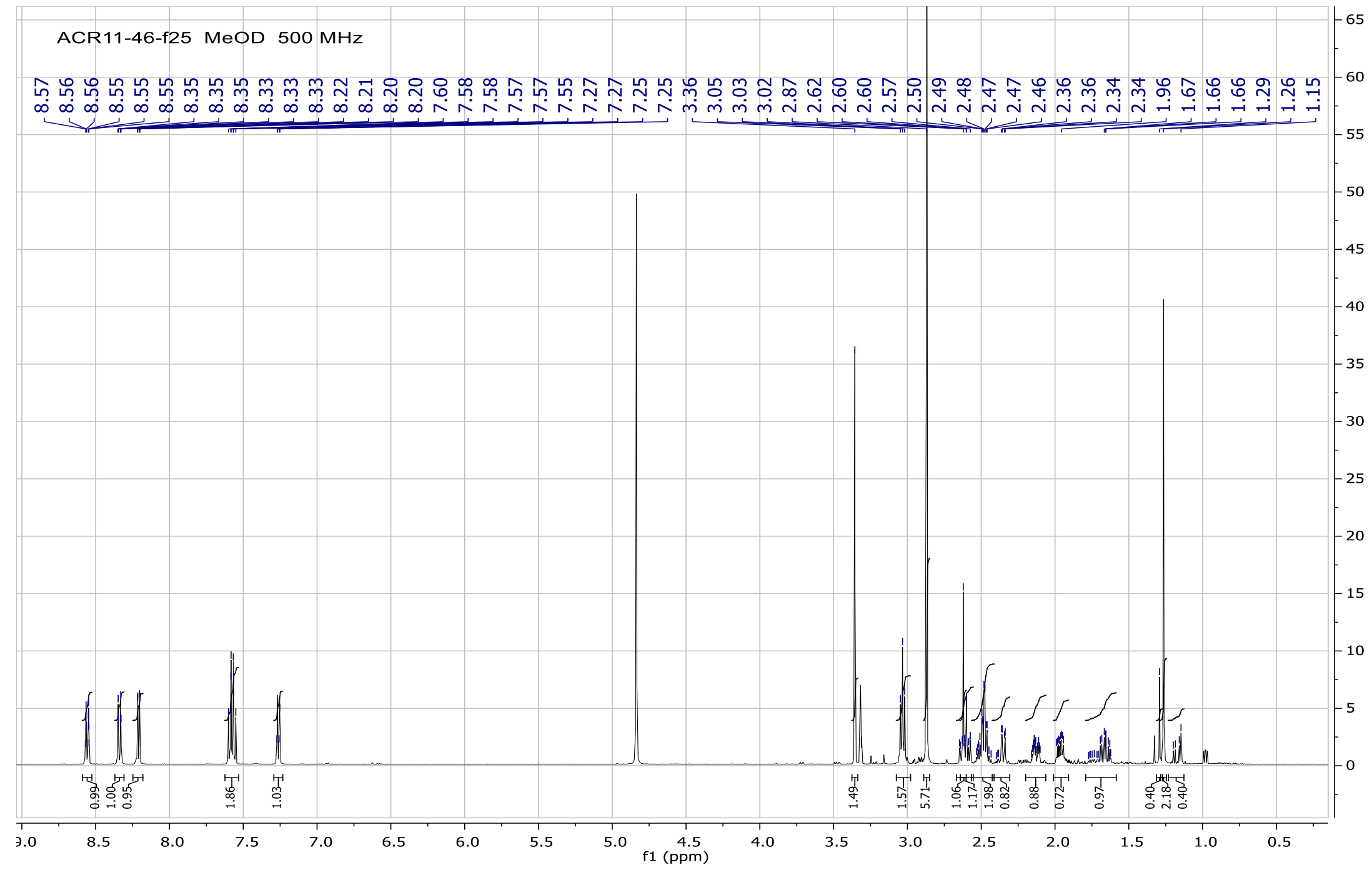


Figure S13. ${ }^{13} \mathrm{C}$ NMR in CDOD (500 MHz) of adduct 2 .

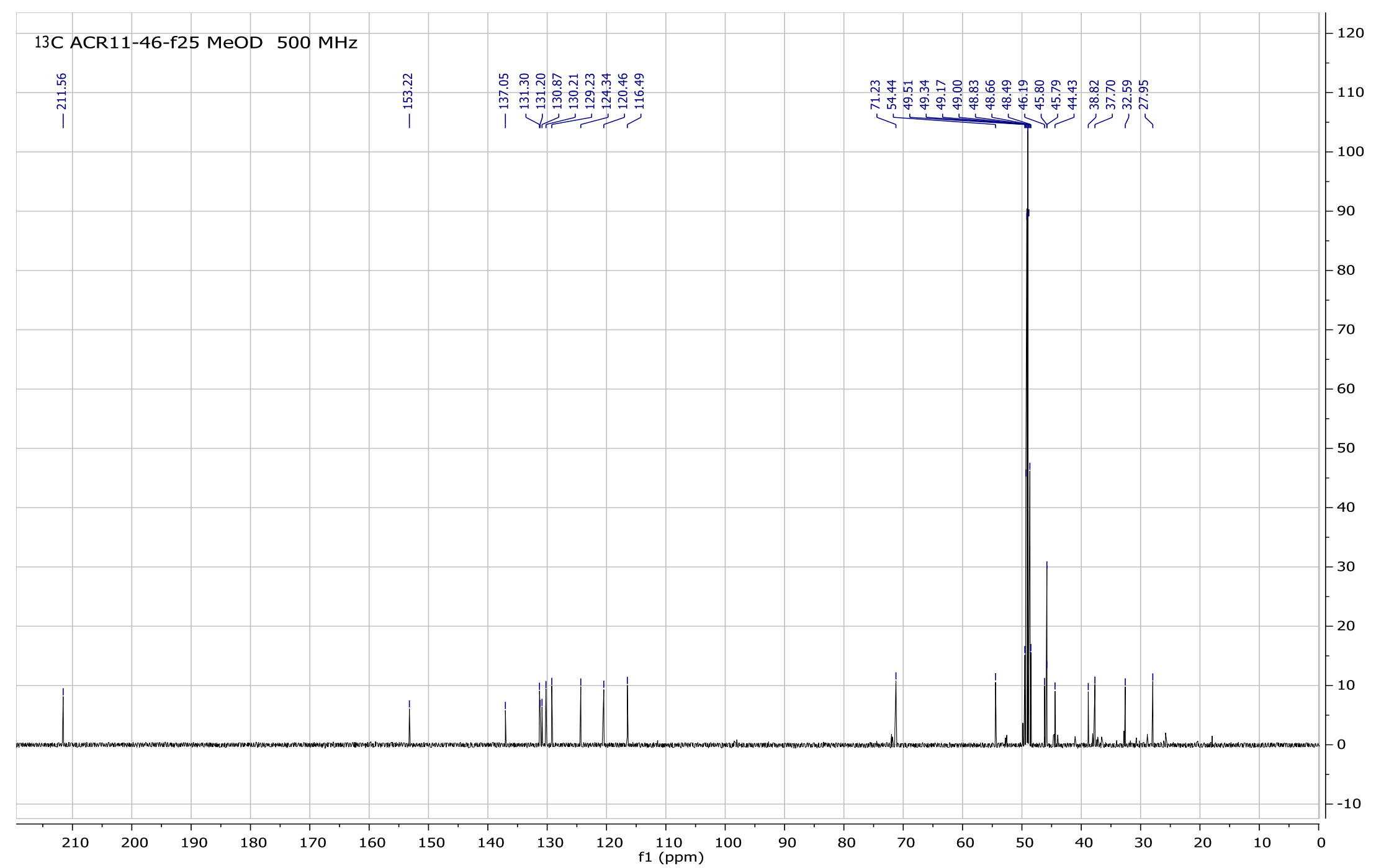

